\title{
Eine vergleichende Kostenanalyse von sechs Anticholinergika zur Behandlung der überaktiven Blase und Inkontinenz in Deutschland
}

\author{
Stefan Felder • Thomas Mayrhofer
}

Online publiziert: 31. Januar 2014

(C) The Author(s) 2014. Dieser Artikel ist auf Springerlink.com mit Open Access verfügbar

\begin{abstract}
Objective: Comparing the costs of solifenacin, oxybutynin, trospium chloride, tolterodine, propiverine, and darifenacin for the treatment of overactive bladder (OAB) and incontinence in Germany.

Data and methods: Costs are assessed from a (public) health insurance perspective. The study is based on a unique sickness fund dataset of 2.9 million insured persons which includes records on outpatient care, inpatient care, pharmaceuticals, adjuvant therapies, and medical aids for 2009. 24,039 persons received anticholinergics and were thus classified as OAB patients. 4,152 of these patients also suffered from incontinence. Multiple linear regression models were performed, controlling for age and gender effects on costs.

Results: OAB patients caused additional costs of $€ 782$ compared to non-OAB patients. Patients treated with propiverine had the lowest additional costs ( $€$ 691) and patients treated with tolterodine the highest $(€ 1,124)$. In the special case of incontinence, patients treated with solifenacin incurred by far the lowest additional costs $(€ 1,320)$, patients treated with tolterodine the highest $(€ 2,198)$. The low treatment costs for solifenacin are mainly driven by lower spending on medical aids, especially due to lower pad usage. All results are highly significant $(p<0.01)$.

Conclusion: OAB patients treated with propiverine and incontinent patients treated with solifenacin have the lowest
\end{abstract}

S. Felder · T. Mayrhofer

CINCH - Gesundheitsökonomisches Zentrum Essen, Universität Duisburg-Essen, Edmund-Körner Platz 2, 45127 Essen, Deutschland

\section{S. Felder $(\varangle)$}

Wirtschaftswissenschaftliche Fakultät, Universität Basel, Peter Merian-Weg 6, 4002 Basel, Schweiz

e-mail: stefan.felder@unibas.ch additional costs. In both patient groups tolterodine is associated with the highest additional cost of treatment.

\section{Einleitung}

Schätzungen gehen davon aus, dass in Deutschland zwischen $12,7 \%$ [1] und 18,0\% [2] der über 40jährigen an einer überaktiven Blase (Overactive Bladder; OAB) leiden. Dies entspricht 6,5 Millionen bis 9,2 Millionen Personen. Irwin et al. [3] gehen in ihrer Untersuchung von 8,0 Millionen betroffenen OAB-Patienten älter als 18 Jahre aus. Klotz et al. [1] schätzen zudem, dass ein Drittel der OAB-Patienten an einer Harninkontinenz leiden. Entgegen der Volksmeinung erkranken Männer ebenso häufig an einer überaktiven Blase wie Frauen. Lediglich in Verbindung mit der Dranginkontinenz ist die Prävalenz deutlich höher als bei Männern. Grundsätzlich treten die Beschwerden der überaktiven Blase in allen Altersgruppen auf; die Wahrscheinlichkeit an einer OAB zu erkranken steigt jedoch mit zunehmendem Alter an [4].

Für Deutschland existieren bisher nur wenige Studien zu den Kosten der OAB. Reeves et al. [5] ermitteln direkte Kosten von rund 950 Millionen Euro für das Jahr 2000 und gehen von einem Kostenanstieg von $29 \%$ bis zum Jahr 2020 aus. Die Hauptkosten machen dabei Inkontinenzeinlagen (600 Millionen Euro) und Arzneimittel (200 Millionen Euro) aus. Klotz et al. [1] schätzen ebenfalls die direkten Kosten der OAB, beziehen jedoch auch Ausgaben für Pflege (Pflegeheime) und für Nebenerkrankungen wie Depression mit ein. Sie kommen zum Ergebnis, dass die Kosten für die OAB in Deutschland bei circa 4 Milliarden Euro liegen, wobei jeweils rund 1,8 Milliarden Euro von der Krankenversicherung sowie der Pflegeversicherung getragen werden. Die Kosten für Arzneimittel schätzen sie auf 80 Millionen Euro, 
lediglich zwei Prozent der Gesamtkosten der OAB. Irwin et al. [3] schätzen die direkten Kosten der OAB ohne Aufwendungen für Pflege auf 1,2 Milliarden Euro. Die Kosten für die Pflege werden mit weiteren 1,6 Milliarden Euro veranschlagt. Für indirekte Kosten, die mit dem Produktionsverlust verbunden sind, ermitteln die Autoren einen Wert von 375 Millionen Euro.

Prävalenz und Kosten weisen somit darauf hin, dass die Bedeutung der OAB in Deutschland mit Volkskrankheiten, wie z. B. Diabetes, gleichzusetzen ist. Primäres Ziel sollte es daher sein, effiziente Behandlungsmethoden für die $\mathrm{OAB}$ zu identifizieren. Ursächliche Therapien sind neben operativen Verfahren die Verhaltenstherapie, die Elektrostimulation sowie die Pharmakotherapie. Bei der Pharmakotherapie werden verschiedene Anticholinergika eingesetzt, die die Wirkung von Acetylcholin an den Muskarinrezeptoren blockieren (Antimuskarinergika). Die in Deutschland verbreiteten Anticholinergika sind Darifenacin, Solifenacin, Tolterodin (alle patentgeschützt) sowie Oxybutynin, Propiverin und Trospiumchlorid (alle generisch). Diese Medikamente gehören insbesondere bei der Behandlung der überaktiven Blase in Verbindung mit Dranginkontinenz zur Standardtherapie $[6,7]$. Die in Deutschland zugelassenen Arzneimittel unterscheiden sich sowohl hinsichtlich der Wirkmechanismen als auch der Wirkstärken. Qualitativ gute und Placebokontrollierte Head-to-Head-Studien zur Effizienz dieser Medikamente sind bisher kaum verfügbar. Die bestehenden Studien zeigen eine annähernd gleiche Wirksamkeit aller verfügbaren Anticholinergika; jedoch ist ihre Patientenverträglichkeit unterschiedlich [8-10]. Studien, die einen Kostenvergleich der Anticholinergika für Deutschland durchführen, fehlen bisher gänzlich.

Diese Lücke soll mit der vorliegenden Kostenvergleichsstudie geschlossen werden. Diese Studie führt, einen Kostenvergleich verschiedener medikamentöser Therapien zur Behandlung der überaktiven Blase und der Inkontinenz in Deutschland aus Sicht der gesetzlichen Krankenkasse durch. Dabei wird überprüft, ob der Einsatz von neuen Wirkstoffen wie Tolterodin, Darifenacin und Solifenacin, die einerseits teurer sind als ältere Wirkstoffe, aber andererseits mit weniger Nebenwirkungen einhergehen [8], insgesamt zu einer Verringerung der Kosten für die Behandlung der überaktiven Blase führen. Zusätzlich werden die Kosten der Behandlung von Patienten mit trockener und nasser OAB (im Folgenden auch Inkontinenz genannt) mit unterschiedlichen OABMedikamenten verglichen.

Das Papier ist wie folgt aufgebaut. Im nächsten Kapitel werden die verwendeten Daten vorgestellt. Im dritten Kapitel wird die Methodik erläutert. Im vierten Kapitel erfolgt die Darstellung und im fünften Kapitel eine Diskussion der Ergebnisse. Das sechste Kapitel fasst zusammen und zieht ein Fazit über die gewonnenen Erkenntnisse.

\section{Daten}

Diese Studie beruht auf mehreren Datensätzen, die von einer großen deutschen Krankenkasse zur Verfügung gestellt wurden. Die Datensätze beziehen sich auf das Jahr 2009 und beinhalten Grunddaten der Versicherten, insbesondere ambulante Daten, stationäre Daten, Arzneimitteldaten, Heilund Hilfsmitteldaten sowie Pflege- und Arbeitsunfähigkeitsdaten. Da die Studie aus Sicht der gesetzlichen Krankenversicherung erfolgt, wurde auf eine Auswertung der Pflegedaten verzichtet. Ferner wurde aufgrund des hohen Durchschnittsalters und der damit einhergehenden Verzerrung hinsichtlich der Erwerbstätigkeit keine Auswertung der Arbeitsunfähigkeitsdaten sowie des Krankengeldes durchgeführt. Alle Datensätze lassen sich mittels anonymisierter Pseudoversichertennummern miteinander verbinden.

Die Datensätze der Krankenkasse umfassen insgesamt 125.896 Versicherte und bestehen aus zwei Stichprobenpopulationen. Aus der Grundgesamtheit der circa 2,9 Mio. Versicherten der Krankenkasse wurden 25.896 identifiziert und als OAB-Patienten klassifiziert, denen im Jahr 2009 ein Anticholinergikum verschrieben wurde (OAB-Patienten wurden somit nur anhand von Arzneimittelverschreibungen klassifiziert.) Zusätzlich wurde eine Zufallsstichprobe von 100.000 Personen ohne OAB aus der Grundgesamtheit der Versicherten gezogen. Diese Zufallsstichprobe umfasst somit rund 3,5\% aller Versicherten dieser Krankenkasse.

Von den rund 25.900 Versicherten, die Arzneimittel gegen OAB erhielten, wiesen 1.857 einen Mehrfachbezug auf, d. h. ihnen wurde im Betrachtungszeitraum mehr als ein OAB-Wirkstoff verschrieben. Diese Patienten wurden aus der Untersuchung ausgeschlossen, da bei Ihnen keine klare Kostenzuordnung zu den einzelnen Medikamenten möglich war. Der Kostenvergleich verschiedener medikamentöser Therapien zur Behandlung der überaktiven Blase in Deutschland bezieht sich somit auf alle 24.039 Versicherte unter OAB-Medikation.

Zusätzlich zum Kostenvergleich in Bezug auf die überaktive Blase wurde ein Kostenvergleich der medikamentösen Therapien für inkontinente Patienten durchgeführt. Die Klassifizierung der Inkontinenzpatienten erfolgte über den Verbrauch an Inkontinenzhilfen im Bereich Hilfsmittel. OAB-Patienten die Inkontinenzhilfen benötigten wurden als inkontinent oder OAB-wet klassifiziert (4.152 Versicherte), Patienten, die keine Inkontinenzhilfen benötigten, als OABdry (19.887 Versicherte). Der Kostenvergleich der Inkontinenzpatienten bezieht sich dabei auf die trockenen OAB Patienten.

Von den 24.039 Versicherten mit OAB-Identifizierung und Einfachbezug wurden rund $38 \%$ mit Trospiumchlorid, $24 \%$ mit Oxybutynin, $13 \%$ mit Propiverin und $12 \%$ mit Solifenacin behandelt. Dies entspricht in etwa den Marktan- 
Tab. 1 Übersicht über Anzahl, Geschlecht und Alter der Versicherten

\begin{tabular}{|c|c|c|c|c|c|c|c|c|c|c|c|c|}
\hline \multirow[t]{2}{*}{ Wirkstoff } & \multicolumn{3}{|c|}{ Patienten } & \multicolumn{3}{|l|}{ Anteil } & \multicolumn{3}{|c|}{ Anteil Männer } & \multicolumn{3}{|c|}{ Durchschnittsalter } \\
\hline & $\begin{array}{l}\text { OAB } \\
\text { dry }\end{array}$ & $\begin{array}{l}\text { OAB } \\
\text { wet }\end{array}$ & $\begin{array}{l}\text { OAB } \\
\text { sum }\end{array}$ & $\begin{array}{l}\text { OAB } \\
\text { dry }\end{array}$ & $\begin{array}{l}\text { OAB } \\
\text { wet }\end{array}$ & $\begin{array}{l}\text { OAB } \\
\text { sum }\end{array}$ & $\begin{array}{l}\text { OAB } \\
\text { dry }\end{array}$ & $\begin{array}{l}\text { OAB } \\
\text { wet }\end{array}$ & $\begin{array}{l}\text { OAB } \\
\text { sum }\end{array}$ & $\begin{array}{l}\text { OAB } \\
\text { dry }\end{array}$ & $\begin{array}{l}\text { OAB } \\
\text { wet }\end{array}$ & $\begin{array}{l}\text { OAB } \\
\text { sum }\end{array}$ \\
\hline Solifenacin & 2.547 & 377 & 2.924 & $13 \%$ & $9 \%$ & $12 \%$ & $24,3 \%$ & $26,5 \%$ & $24,6 \%$ & 66,9 & 74,7 & 67,9 \\
\hline Oxybutynin & 4.777 & 1.061 & 5.838 & $24 \%$ & $26 \%$ & $24 \%$ & $33,0 \%$ & $34,3 \%$ & $33,2 \%$ & 63,0 & 69,7 & 64,2 \\
\hline Trospium-Chlorid & 7.521 & 1.688 & 9.209 & $38 \%$ & $41 \%$ & $38 \%$ & $31,4 \%$ & $34,3 \%$ & $31,9 \%$ & 64,3 & 75,6 & 66,4 \\
\hline Tolterodin & 1.299 & 325 & 1.624 & $7 \%$ & $8 \%$ & $7 \%$ & $23,6 \%$ & $25,8 \%$ & $24,1 \%$ & 69,0 & 74,5 & 70,1 \\
\hline Propiverin & 2.581 & 428 & 3.009 & $13 \%$ & $10 \%$ & $13 \%$ & $41,2 \%$ & $39,5 \%$ & $40,9 \%$ & 48,1 & 56,3 & 49,3 \\
\hline Darifenacin & 1.162 & 273 & 1.435 & $6 \%$ & $7 \%$ & $6 \%$ & $34,2 \%$ & $31,9 \%$ & $33,7 \%$ & 66,8 & 73,7 & 68,1 \\
\hline OAB-Patienten & 19.887 & 4.152 & 24.039 & $100 \%$ & $100 \%$ & $100 \%$ & $31,8 \%$ & $33,3 \%$ & $32,1 \%$ & 62,7 & 71,8 & 64,2 \\
\hline Nicht-OAB-Patienten & 100.000 & & & - & & & $49,1 \%$ & & & 41,5 & & \\
\hline
\end{tabular}

Tab. 2 Marktanteil Harninkontinenzprodukte in Prozent für das Jahr 2009

${ }^{a}$ Duloxetine hat lediglich die Zulassung zur Anwendung bei Belastungsinkontinenz, nicht jedoch zur Behandlung der überaktiven Blase bzw. Dranginkontinenz Quelle: IMS Health Care Germany

\begin{tabular}{llcc}
\hline Wirkstoffe & $\begin{array}{l}\text { Marktanteil nach } \\
\text { Umsatz }\end{array}$ & $\begin{array}{l}\text { Marktanteil nach } \\
\text { Verordnungen }\end{array}$ & $\begin{array}{c}\text { Marktanteil nach } \\
\text { Zähleinheiten }\end{array}$ \\
\hline Solifenacin & 25,3 & 13,2 & 12,4 \\
Trospium & 20,8 & 35,1 & 36,0 \\
Tolterodin & 15,4 & 9,6 & 9,9 \\
Propiverin & 13,1 & 12,4 & 13,2 \\
Darifenacin $^{\text {Duloxetine }}$ & 10,6 & 5,4 & 5,2 \\
Oxybutynin $^{\text {a }}$ & 5,7 & 3,0 & 3,0 \\
Fesoterodine & 5,3 & 18,6 & 18,1 \\
Flavoxate & 3,7 & 2,4 & 1,8 \\
\hline
\end{tabular}

teilen an Verordnungen im Zeitraum 2009, wobei Trospiumchlorid und Oxybutynin in diesem Datensatz überrepräsentiert sind (siehe Tab. 1 und 2).

Tabelle 1 zeigt, dass es zwischen den Empfängern der verschiedenen Medikamente zum Teil erhebliche Unterschiede hinsichtlich des Geschlechts (Bsp.: Propiverin 40,9 \% Männer vs. Tolterodin 24,1 \% Männer) und des durchschnittlichen Alters (Bsp.: Propiverin 49,3 Jahre vs. Tolterodin 70,1 Jahre) gibt. Zudem zeigt sich, dass inkontinente Patienten im Durchschnitt 9 Jahre älter sind als Patienten mit trockener OAB.

\section{Methodik}

Der Kostenvergleich erfolgte mit der Methode der multiplen linearen Regressionsanalyse angewendet. Diese ermöglicht es, unterschiedliche Einflüsse auf die Kostenschätzung zu kontrollieren. Da sich im vorliegenden Fall sowohl das durchschnittliche Alter als auch der Anteil der Männer zwischen den verschiedenen Stichproben unterscheiden, muss berücksichtigt werden, wenn Kostenunterschiede zwischen den Gruppen teilweise hierauf zurückzuführen sind.
Die angewandten Regressionsmodelle sehen daher wie folgt aus:

$$
\begin{aligned}
y_{i}= & \beta_{0}+\beta_{1} \cdot \text { OAB }_{i}+\beta_{2} \cdot \text { Alter }_{i}+\beta_{3} \cdot\left(\text { Alter }_{i}\right)^{2} \\
& +\beta_{4} \cdot \text { Männlich }_{i}+\beta_{5} \cdot\left(\text { Alter }_{i} \cdot \text { Männlich }_{i}\right)+u_{i}
\end{aligned}
$$

und

$$
\begin{aligned}
y_{i}= & \gamma_{0}+\gamma_{1, j} \cdot x_{j i}+\gamma_{2} \cdot \text { Alter }_{i}+\gamma_{3} \cdot\left(\text { Alter }_{i}\right)^{2} \\
& +\gamma_{4} \cdot \text { Männlich }_{i}+\gamma_{5} \cdot\left(\text { Alter }_{i} \cdot \text { Männlich }_{i}\right)+e_{i},
\end{aligned}
$$

wobei $y_{i}$ die abhängige oder zu erklärende Variable darstellt ( $i=$ Beobachtung), die im Ergebnisteil als Spaltenkopf in den Tabellen gekennzeichnet ist. Die Größen $\beta_{0}$ und $\gamma_{0}$ kennzeichnen die Regressionskonstanten, $\beta$ und $\gamma$ die Regressionskoeffizienten der unabhängigen oder erklärenden Variablen und $u$ bzw. $e$ die Residuen. Die Variable $O A B_{i}$ nimmt den Wert 1 an, wenn der Versicherte an $\mathrm{OAB}$ leidet, und den Wert 0, wenn dies nicht der Fall ist. Der dazugehörige Regressionskoeffizient gibt die zusätzlichen Kosten an, die ein OAB-Patient im Vergleich zu einem nicht-OAB-Patienten verursacht. Die Variable $x_{j i}$ wird verwendet, um zu bestimmen, welche zusätzlichen Kosten ein OAB-Patient verursacht, der mit dem Wirkstoff $(j)$ behandelt wird. Die Regressionskoeffizienten für OAB-Patienten 
insgesamt sowie für die Behandlung mit den einzelnen Wirkstoffen bilden die Grundlage für die Tabellen im Ergebnisteil.

Das Alter eines Versicherten wird unter Annahme eines nicht-linearen Funktionsverlaufs (Alter und Alter ${ }^{2}$ ) einbezogen. Geschlecht wird sowohl als binäre Variable als auch als Interaktionsterm zusammen mit dem Alter berücksichtigt. Regressionskoeffizienten zu Alter und Geschlecht werden in den Ergebnistabellen nicht ausgewiesen, da sie lediglich der Standardisierung, d. h. der Herausrechnung etwaiger Altersund Geschlechtseffekte, dienen.

Um den Anteil an OAB-Patienten im Bezug auf die Grundgesamtheit der Versicherten wiederzugeben wurden nicht-OAB-Patienten - die auf einer 3,5\%-igen Stichprobe der Grundgesamtheit beruhen - in den Regressionen mit dem Faktor 29 gewichtet. Zudem erfolgte die Verwendung von robusten Standardfehlern, um für Heteroskedastizität zu kontrollieren.

Für die Auswertung der Inkontinenzpatienten wurden die gleichen Regressionsmodelle genutzt.

\section{Ergebnisse}

Ein Problem des Kostenvergleichs auf Basis der Gesamtkosten liegt in der möglicherweise falschen Berücksichtigung von Nebenerkrankungen der OAB, wie z. B. Diabetes oder Herzversagen [11], bei denen eine kausale Beziehung jedoch nicht belegt werden kann [12]. In dieser Studie wird daher ein spezifischer Kostenvergleich durchgeführt, der sich auf Ausgaben für diejenigen Begleiterscheinungen beschränkt, die laut Literatur mit der OAB weitestgehend in Verbindung gebracht werden können [13]. Dies sind vor allem Ausgaben für die folgenden Begleit- und Folgeerscheinungen: Harnwegsinfektionen, Hautprobleme und -infektionen, Stürze und Frakturen, Depression sowie Vulvovaginits [5, 11, 14].

\subsection{Stationäre Kosten}

Als erstes wurde untersucht, wie sich die Ausgaben für die stationäre Behandlung der häufigsten Begleiterscheinungen zwischen den Empfängern der einzelnen OABWirkstoffgruppen unterscheiden. Einige dieser Begleiterscheinungen sind auf die Nebenwirkungen einzelner OABMedikamente zurückzuführen. So können Frakturen einerseits aufgrund von OAB-bedingten Stürzen (schneller bis hektischer Gang zur Toilette), andererseits auch aufgrund von medikamentenbedingten Stürzen (Sehstörungen und Schwindelgefühl insbesondere bei älteren OABMedikamenten) [9, 15] entstehen.

Die Zuordnung der Begleiterscheinungen zu OAB- sowie Nicht-OAB-Patienten wurde anhand der ICD-Codes
Tab. 3 ICD-Codes der Nebenerkrankungen/Folgeerscheinungen der $\mathrm{OAB}$

\begin{tabular}{ll}
\hline Folgeerscheinungen & ICD 10 \\
\hline $\begin{array}{l}\text { Stürze und Frakturen } \\
\text { Harnwegsinfektionen und }\end{array}$ & S32, S42, S52, S62, S72, S82 \\
OAB-Indikation & R32, R35, N39, N32.8 \\
Hautinfektionen & L08 \\
Vulvovaginitis & N76 \\
Depressionen & F33 \\
\hline
\end{tabular}

der Enddiagnosen vorgenommen (siehe Tab. 3). Zusätzlich erfolgte eine Berücksichtigung der Ausgaben im Bereich Harnwegsinfektionen.

Im stationären Sektor unterschieden sich OAB-Patienten von Nicht-OAB-Patienten hinsichtlich der Folgeerkrankungen lediglich bei Frakturen, Harnwegsinfektionen sowie Depression (siehe Tab. 4). Während Solifenacin- und Darifenacin-Patienten nicht signifikant mehr Frakturen erlitten als Nicht-OAB-Patienten, weisen insbesondere Patienten die mit Tolterodin behandelt wurden, erhöhte Kosten in diesem Bereich auf. Dies gilt auch für den Bereich der Harnwegsinfektionen sowie für Depression. Bei Hautinfektionen und Vulvovaginitis gab es hingegen nur zwischen Trospiumchlorid- bzw. Propiverin-Patienten und NichtOAB-Patienten leichte (aber signifikante) Unterschiede. Insgesamt weisen Tolterodin-Patienten mit Abstand die höchsten zusätzlichen Kosten im stationären Bereich auf. Dagegen verursachten Propiverin- und Solifenacin-Patienten die geringsten zusätzlichen Ausgaben im stationären Bereich.

Schränkt man die Untersuchung auf Inkontinenzpatienten ein, zeigt sich ein ähnliches Bild. Tolterodin-Patienten weisen die höchsten Kosten im Vergleich zu Patienten mit trockener OAB auf. Die geringsten Ausgaben verursachen dagegen Solifenacin-Patienten. Insbesondere die Ausgaben für Frakturen sind bei Solifenacin-Patienten niedriger als unter den anderen Anticholinergika. Dies deutet auf eine bessere Wirksamkeit dieses Wirkstoffes im Sinne geringerer Nebenwirkungen im Bereich Stürze. Auch bezüglich Depression weisen Solifenacin-Patienten neben DarifenacinPatienten die geringsten Kosten auf. Auch hier könnte eine höhere Wirksamkeit der Medikamente (bezogen auf Inkontinenz) zu einem aktiverem Leben und damit zu höherer Lebensqualität und weniger Depressionsanfälligkeit führen. Insgesamt unterscheiden sich Inkontinenzpatienten hinsichtlich der Ausgaben für Depressionen jedoch nicht signifikant von Patienten mit trockener OAB.

\subsection{Ambulanter Bereich (Arztkosten)}

Auch im ambulanten Bereich (Arztkosten) wurden die häufigsten Begleiterscheinungen als Grundlage für den spezifischen Kostenvergleich gewählt. Eine Kostenzuordnung 
Tab. 4 Zusätzliche Kosten von OAB- und INK-Patienten in Euro: Stationärer Bereich

\begin{tabular}{|c|c|c|c|c|c|c|}
\hline Wirkstoff & Frakturen & $\begin{array}{l}\text { Harnwegsinf. } \\
\text { und } O A B\end{array}$ & Hautinfektionen & Vulvovaginitis & Depressionen & Gesamt \\
\hline \multicolumn{7}{|l|}{ Zusätzliche Kosten $O A B$} \\
\hline Solifenacin & 8,15 & $46,98^{* * *}$ & $-0,04^{* * *}$ & 0,23 & 12,32 & $67,64^{* * *}$ \\
\hline Oxybutynin & $20,64^{*}$ & $32,76^{* * *}$ & $-0,06^{* * *}$ & 0,05 & $17,43^{*}$ & $70,81^{* * * *}$ \\
\hline Trospium-Chlorid & $40,95^{* * *}$ & $39,25^{* * *}$ & $0,57^{*}$ & 0,14 & 8,63 & $89,54^{* * *}$ \\
\hline Tolterodin & $96,91^{* * *}$ & $69,43^{* * *}$ & $-0,03^{* * *}$ & $-0,29^{* * *}$ & 26,79 & $192,80^{* * * *}$ \\
\hline Propiverin & 14,32 & $27,60^{* * *}$ & $-0,12^{* * *}$ & 1,27 & $21,99^{*}$ & $65,07^{* * *}$ \\
\hline Darifenacin & 14,22 & $74,64^{* * *}$ & $-0,06^{* * *}$ & $-0,24^{* * *}$ & 1,68 & $90,24^{* * *}$ \\
\hline OAB-Patienten gesamt & $30,86^{* * *}$ & $41,29^{* * *}$ & 0,18 & 0,22 & $13,70^{* * *}$ & $86,25^{* * *}$ \\
\hline \multicolumn{7}{|l|}{ Zusätzliche Kosten INK } \\
\hline Solifenacin & 6,48 & $102,16^{* * *}$ & 0,03 & 3,31 & $-17,65^{* * *}$ & 94,33 \\
\hline Oxybutynin & $71,89^{* *}$ & $98,79^{* * *}$ & $-0,02$ & $-0,54$ & 50,83 & $220,96^{* * *}$ \\
\hline Trospium-Chlorid & $82,74^{* * *}$ & $110,02^{* * *}$ & 2,13 & 0,42 & 9,82 & $205,13^{* * * *}$ \\
\hline Tolterodin & $196,94^{*}$ & $219,89^{* * *}$ & 0,05 & $-0,60$ & 51,74 & $468,02^{* * * *}$ \\
\hline Propiverin & $112,25^{*}$ & $109,95^{* *}$ & 0,00 & $-0,65$ & $-11,58^{* * *}$ & $209,97^{* * * *}$ \\
\hline Darifenacin & 26,02 & $163,95^{* *}$ & $-0,03$ & $-0,50$ & $-18,40^{* * * *}$ & $171,04^{*}$ \\
\hline INK-Patienten gesamt & $81,24^{* * *}$ & $118,52^{* * *}$ & 0,85 & 0,18 & 17,01 & $217,80^{\text {**** }}$ \\
\hline
\end{tabular}

$\mathrm{OAB}=$ überaktive Blase; INK = Inkontinenz

${ }^{*} p<0,10 ;{ }^{* *} p<0,05 ;{ }^{* * *} p<0,01$; robuste Standardfehler

zu einzelnen Behandlungsepisoden konnte nur indirekt erfolgen, da die ambulanten Abrechnungen nur nach Versicherten, Quartal und Facharztgruppe differenziert vorlagen. Wies ein Versicherter in einem Quartal beispielsweise eine Fraktur auf, so werden in dieser Analyse die Quartalsausgaben in der entsprechenden Facharztgruppe (z. B. Orthopädie) als Behandlungskosten zugrundegelegt.

Insgesamt sind die Kosten deutlich homogener über die Wirkstoffe verteilt als im stationären Bereich (siehe Tab. 5). Die geringsten zusätzlichen ambulanten Kosten weisen Propiverin-Patienten auf, die höchsten haben Patienten, die mit Darifenacin behandelt wurden. Dies gilt sowohl für OAB- als auch für Inkontinenzpatienten.

Die zusätzlichen Kosten für Frakturen sind im ambulanten Bereich deutlich geringer, jene für Harnwegsinfektionen deutlich höher als im stationären Bereich. Zusätzliche Ausgaben für Hautinfektionen und Vulvovaginitis sind im ambulanten Bereich statistisch signifikant, jedoch aus ökonomischer Sicht als eher unbedeutend einzustufen.

\subsection{Arzneimittelkosten}

Hinsichtlich der Arzneimittelkosten wurde zwischen zusätzlichen Ausgaben für die OAB-Präparate selbst, Ausgaben aufgrund ihrer Nebenwirkungen sowie Arzneimittelausgaben aufgrund von Folgeerscheinungen der OAB unterschieden. Nebenwirkungen der OAB-Medikation beinhalten Verstopfung (NW1), Herzrhythmusstörungen (NW2) sowie
Übelkeit und Erbrechen, wobei die Ergebnisse der Regressionsschätzung für letzteres aufgrund nichtsignifikanter Ergebnisse in Tab. 6 nicht abgebildet sind. Bei den Folgeerscheinungen wurden zusätzliche Arzneimittelausgaben aufgrund von Frakturen (FE1) sowie von Depression (FE2) untersucht. Im Unterschied zum stationären und ambulanten Bereich erfolgt die Zuordnung nicht über ICD-Nummern, sondern über die ATC-Klassifikation (siehe hierzu Tab. 6).

Auffällig sind vor allem die hohen Ausgabenunterschiede bei der OAB-Medikation. Während OAB-Patienten die mit Oxybutynin behandelt wurden die geringsten Kosten aufweisen, verursachen Solifenacin-Patienten und TolterodinPatienten die höchsten Ausgaben (siehe Tab. 7). Dies deckt sich mit den Marktanteilen der Arzneimittel: Während Solifenacin von allen Harninkontinenzprodukten mit 25,3\% den höchsten Marktanteil nach Umsatz besitzt (bei 13,2 \% Marktanteil nach Verordnungen), weist Oxybutynin lediglich einen Marktanteil nach Umsatz von 5,3\% auf (bei 18,6\% Marktanteil nach Verordnungen). Das Ergebnis spiegelt sich auch in den zusätzlichen Gesamtausgaben im Bereich Arzneimittel wider. Ohne Berücksichtigung der OABPräparate weisen Patienten, die mit Solifenacin oder Propiverin behandelt wurden, zwar die geringsten zusätzlichen Arzneimittelausgaben auf. Bezieht man jedoch die Kosten für die OAB-Präparate selbst mit ein, verursachen Solifenancin-Patienten die dritthöchsten Arzneimittelausgaben. 
Tab. 5 Zusätzliche Arztkosten von OAB- und INK-Patienten in Euro

\begin{tabular}{|c|c|c|c|c|c|c|}
\hline Wirkstoff & Frakturen & $\begin{array}{l}\text { Harnwegsinf. } \\
\text { und } O A B\end{array}$ & Hautinfektionen & Vulvovaginitis & Depressionen & Gesamt \\
\hline \multicolumn{7}{|l|}{ Zusätzliche Kosten $O A B$} \\
\hline Solifenacin & 2,12 & $123,00^{* * *}$ & $0,56^{* *}$ & $4,09^{* * *}$ & $16,88^{* * *}$ & $146,65^{* * *}$ \\
\hline Oxybutynin & $8,09^{* * *}$ & $119,60^{* * *}$ & $0,48^{* * *}$ & $3,42^{* * *}$ & $9,40^{* * *}$ & $141,12^{* * *}$ \\
\hline Trospium-Chlorid & $7,85^{* * *}$ & $112,75^{* * *}$ & $0,58^{* * *}$ & $4,06^{* * *}$ & $9,82^{* * *}$ & $135,01^{* * *}$ \\
\hline Tolterodin & $10,82^{* * *}$ & $127,72^{* * *}$ & 0,39 & $2,80^{* * * *}$ & $8,51^{* * * *}$ & $150,24^{* * * *}$ \\
\hline Propiverin & $3,80^{* * *}$ & $114,42^{* * *}$ & $0,37^{*}$ & $2,36^{* * *}$ & $10,10^{* * *}$ & $131,07^{* * *}$ \\
\hline Darifenacin & $9,56^{* * *}$ & $139,73^{* * *}$ & $-0,01$ & $2,92^{* * *}$ & $15,80^{* * *}$ & $168,00^{* * *}$ \\
\hline OAB-Patienten gesamt & $7,01^{* * * *}$ & $118,49^{* * *}$ & $0,48^{* * *}$ & $3,54^{* * *}$ & $10,88^{* * *}$ & $140,40^{* * *}$ \\
\hline \multicolumn{7}{|l|}{ Zusätzliche Kosten INK } \\
\hline Solifenacin & 1,65 & $110,49^{* * *}$ & 1,22 & $-0,21$ & $13,62^{* *}$ & $126,76^{\text {*** }}$ \\
\hline Oxybutynin & $18,57^{* * *}$ & $112,36^{* * *}$ & $1,45^{* *}$ & $-0,69$ & $7,35^{* *}$ & $139,04^{* * *}$ \\
\hline Trospium-Chlorid & $22,50^{* * * *}$ & $102,48^{* * *}$ & $1,89^{* * *}$ & $-1,62^{* * *}$ & $4,83^{*}$ & $130,09^{* * * *}$ \\
\hline Tolterodin & $10,38^{*}$ & $98,82^{* * *}$ & 0,11 & $-1,80^{* *}$ & 2,29 & $109,80^{* * * *}$ \\
\hline Propiverin & 2,61 & $102,21^{* * *}$ & $-0,06$ & $-0,99$ & $-0,77$ & $102,99^{* * * *}$ \\
\hline Darifenacin & $36,30^{* * *}$ & $129,75^{* * *}$ & $-0,41$ & 0,29 & $12,97^{*}$ & $178,91^{* * *}$ \\
\hline INK-Patienten gesamt & $17,46^{* * *}$ & $107,25^{* * *}$ & $1,22^{* * *}$ & $-1,07^{* * *}$ & $6,03^{* * *}$ & $130,89^{* * *}$ \\
\hline
\end{tabular}

Angenommener Entgeltpunkt von 3,5 Cent. $\mathrm{OAB}=$ überaktive Blase; INK = Inkontinenz

${ }^{*} p<0,10 ;{ }^{* *} p<0,05 ;{ }^{* * *} p<0,01$; robuste Standardfehler

Tab. 6 ATC-Codes der Nebenwirkungen/ Folgeerscheinungen der $\mathrm{OAB}$ und OAB-Medikamente

\begin{tabular}{lll}
\hline $\begin{array}{l}\text { Folgeerscheinungen und } \\
\text { Medikamentennebenwirkungen } \\
\text { der OAB }\end{array}$ & $\begin{array}{l}\text { Arzneimittelkategorie gegen } \\
\text { Folgeerkrankungen und } \\
\text { Nebenwirkungen }\end{array}$ & ATC-Einteilung \\
\hline Frakturen & Analgetika & N02 \\
Depressionen & Psychoanaleptika & N06 \\
Herzrhythmusstörungen & $\begin{array}{l}\text { Antithrombotische Mittel, Herztherapie, } \\
\text { Beta-Adrenorezeptoren-Antagonisten }\end{array}$ & B01, C01, C07 \\
Verstopfung & Laxanzien & A06 \\
Übelkeit und Erbrechen & Antiemetika und Mittel gegen Übelkeit & A04 \\
\hline
\end{tabular}

Hinsichtlich der Ausgaben aufgrund von Nebenwirkungen und Folgeerscheinungen zeigt sich für die einzelnen Wirkstoffe ein vergleichsweise homogenes Bild. Die größten Unterschiede bestehen bei Schmerzmitteln für Frakturen, wobei hier Solifenacin-Patienten und PropiverinPatienten die geringsten und Darifenacin-Patienten die höchsten zusätzlichen Kosten verursachen. Auch hier deutet dies (zusammen mit den Ergebnissen aus dem ambulanten und stationären Bereich) auf eine höhere Wirksamkeit bezüglich des Sturzrisikos hin.

Auch Inkontinenzpatienten, die mit Oxybutynin behandelt wurden, weisen die geringsten und Patienten, die mit Tolterodin oder Solifenancin behandelt wurden, die höchsten zusätzlichen Kosten gegenüber Patienten mit trockener OAB auf. Auffallend ist zudem, dass inkontinente Oxybutynin-Patienten signifikant geringere OAB-Medikationskos- ten verursachen als Oxybutynin-Patienten mit trockener OAB.

\subsection{Heilmittelkosten}

Im vorliegenden Datensatz sind Heilmittel in fünf Kategorien eingeteilt: Ergotherapie, Logopädie, Physiotherapie, Podologie und sonstige Heilmittel. Insgesamt weisen OABPatienten rund $€ 181$ zusätzliche Ausgaben im Bereich Heilmittel auf. Der Großteil, rund $€ 134$, beruht dabei auf dem Posten Physiotherapie (siehe Tab. 8). Dies ist auch die einzige Heilmittelart, bei der zumindest mittelbar von einem kausalen Zusammenhang mit OAB-Nebenwirkungen ausgegangen werden kann. Aus diesem Grund berücksichtigt die Analyse auch nur diese zusätzlichen Kosten (siehe Tab. 10).

Für Physiotherapie weisen OAB-Patienten, die mit Solifenacin behandelt wurden, mit rund $€ 97$ die geringsten 
Tab. 7 Zusätzliche Kosten von OAB- und INK-Patienten in Euro: Arzneimittel

\begin{tabular}{|c|c|c|c|c|c|c|c|}
\hline \multirow[t]{2}{*}{ Wirkstoff } & \multirow[t]{2}{*}{ OAB-Med. } & \multicolumn{2}{|c|}{$\begin{array}{l}\text { Zusätzl. Kosten } \\
\text { aufgrund von NW }\end{array}$} & \multicolumn{2}{|c|}{$\begin{array}{l}\text { Zusätzl. Kosten } \\
\text { aufgrund von FE }\end{array}$} & \multicolumn{2}{|l|}{ Gesamt } \\
\hline & & NW1 & NW2 & FE1 & FE2 & Ohne OAB Med. & Mit OAB Med. \\
\hline \multicolumn{8}{|l|}{ Zusätzliche Kosten $O A B$} \\
\hline Solifenacin & $347,27^{* * *}$ & $2,10^{* * * *}$ & $17,75^{* * *}$ & $31,11^{* * *}$ & $29,51^{* * *}$ & $80,48^{* * *}$ & $427,75^{* * *}$ \\
\hline Oxybutynin & $87,21^{* * *}$ & $4,09^{* * * *}$ & $11,37^{* * *}$ & $50,13^{* * *}$ & $29,55^{* * *}$ & $95,15^{* * *}$ & $182,36^{* * *}$ \\
\hline Trospium-Chlorid & $129,45^{* * *}$ & $3,77^{* * *}$ & $13,77^{* * *}$ & $63,09^{* * *}$ & $34,80^{* * *}$ & $115,43^{* * *}$ & $244,88^{* * * *}$ \\
\hline Tolterodin & $351,51^{* * *}$ & $4,37^{* * * *}$ & $18,44^{* * *}$ & $51,00^{* * *}$ & $31,01^{* * *}$ & $104,82^{* * *}$ & $456,33^{* * *}$ \\
\hline Propiverin & $211,81^{* * *}$ & $3,73^{* * *}$ & $12,80^{* * *}$ & $31,56^{* * *}$ & $23,90^{* * *}$ & $71,99^{* * *}$ & $283,80^{* * *}$ \\
\hline Darifenacin & $324,98^{* * *}$ & $4,44^{* * * *}$ & $23,41^{* * *}$ & $77,68^{* * *}$ & $41,82^{* * *}$ & $147,34^{* * *}$ & $472,32^{* * *}$ \\
\hline OAB-Patienten gesamt & $182,63^{* * *}$ & $3,72^{* * *}$ & $14,44^{* * *}$ & $52,15^{* * *}$ & $31,68^{* * *}$ & $101,99^{* * *}$ & $284,60^{* * * *}$ \\
\hline \multicolumn{8}{|l|}{ Zusätzliche Kosten INK } \\
\hline Solifenacin & $222,21^{* * *}$ & $11,52^{* * *}$ & $19,86^{* *}$ & $94,42^{* * *}$ & $66,09^{* * *}$ & $191,89^{* * *}$ & $414,10^{* * *}$ \\
\hline Oxybutynin & $-47,84^{* * *}$ & $13,47^{* * *}$ & $13,47^{* *}$ & $62,67^{* * *}$ & $53,95^{* * *}$ & $143,56^{* * *}$ & $95,72^{* * *}$ \\
\hline Trospium-Chlorid & $9,02^{* *}$ & $11,52^{* * *}$ & $15,39^{* * *}$ & $130,80^{* * *}$ & $52,34^{* * *}$ & $210,06^{* * *}$ & $219,08^{* * *}$ \\
\hline Tolterodin & $216,06^{* * *}$ & $13,86^{* * *}$ & $14,72^{*}$ & $55,56^{* *}$ & $65,53^{* * *}$ & $149,67^{* * *}$ & $365,73^{* * *}$ \\
\hline Propiverin & $144,51^{* * *}$ & $10,11^{* * *}$ & 5,89 & $41,61^{* *}$ & $61,33^{* * *}$ & $118,96^{* * *}$ & $263,46^{* * *}$ \\
\hline Darifenacin & $228,19^{* * * *}$ & $13,05^{* * *}$ & $43,86^{* * *}$ & $118,58^{* *}$ & $82,82^{* * *}$ & $258,32^{* * * *}$ & $486,51^{* * * *}$ \\
\hline INK-Patienten gesamt & $58,71^{* * *}$ & $12,16^{* * *}$ & $16,14^{* * *}$ & $93,83^{* * *}$ & $58,00^{* * *}$ & $180,13^{* * *}$ & $238,84^{* * *}$ \\
\hline
\end{tabular}

$\mathrm{OAB}=$ überaktive Blase; INK = Inkontinenz; NW = Nebenwirkung; FE = Folgeerkrankung; NW1 = Verstopfung; NW2 = Herz-RhythmusStörungen; FE1 = Frakturen/Knochenbrüche; FE2 = Depressionen

a Keine ökonomisch signifikanten Effekte (marginale Effekte liegen bei rund 1 Cent) für Mittel gegen „Übelkeit und Erbrechen“ (ATC: A04) ${ }^{*} p<0,10 ;{ }^{* *} p<0,05 ;{ }^{* * *} p<0,01$; robuste Standardfehler

Tab. 8 Zusätzliche Kosten von $\mathrm{OAB}$ - und INK-Patienten in Euro: Heilmittel
$\mathrm{OAB}=$ überaktive Blase; INK

$=$ Inkontinenz

${ }^{*} p<0,10 ;{ }^{* *} p<0,05$;

${ }^{* * *} p<0,01$; robuste

Standardfehler

\begin{tabular}{lllll}
\hline Wirkstoff $\quad$ Ergotherapie Logopädie Physiotherapie Podologie & $\begin{array}{l}\text { Sonstige } \\
\text { Heilmittel }\end{array}$
\end{tabular}

\begin{tabular}{lcccccc}
\hline Zusätzliche Kosten OAB & & & & & & \\
Solifenacin & $17,57^{* * *}$ & $4,98^{* *}$ & $96,68^{* * *}$ & $3,15^{* * *}$ & $1,29^{* *}$ & $123,68^{* * *}$ \\
Oxybutynin & $29,04^{* * * *}$ & $13,97^{* * *}$ & $146,64^{* * *}$ & $2,79^{* * *}$ & $1,89^{* * *}$ & $194,33^{* * *}$ \\
Trospium-Chlorid & $29,34^{* * *}$ & $12,15^{* * *}$ & $145,34^{* * *}$ & $2,94^{* * *}$ & $3,27^{* * *}$ & $193,05^{* * *}$ \\
Tolterodin & $24,45^{* * *}$ & $7,94^{* *}$ & $142,20^{* * *}$ & $3,27^{* * *}$ & $1,94^{* *}$ & $179,79^{* * *}$ \\
Propiverin & $42,09^{* * *}$ & $22,90^{* * *}$ & $98,61^{* * *}$ & $1,72^{* * *}$ & 0,43 & $165,75^{* * *}$ \\
Darifenacin & $30,80^{* * *}$ & $10,73^{* *}$ & $157,88^{* * *}$ & $4,64^{* * *}$ & $2,19^{*}$ & $206,24^{* * *}$ \\
OAB-Patienten gesamt & $29,20^{* * *}$ & $12,70^{* * *}$ & $134,40^{* * *}$ & $2,90^{* * *}$ & $2,18^{* * *}$ & $181,39^{* * *}$ \\
& & & & & & \\
Zusätzliche Kosten INK & & & & & \\
Solifenacin & $39,57^{* *}$ & $26,17^{* *}$ & $223,77^{* * *}$ & 4,06 & $9,01^{* *}$ & $302,58^{* * *}$ \\
Oxybutynin & $75,32^{* * *}$ & $56,56^{* * *}$ & $421,38^{* * *}$ & $4,82^{* * *}$ & $13,62^{* * *}$ & $571,69^{* * *}$ \\
Trospium-Chlorid & $129,04^{* * *}$ & $69,24^{* * *}$ & $404,93^{* * *}$ & $6,49^{* * *}$ & $19,69^{* * *}$ & $629,40^{* * *}$ \\
Tolterodin & $89,27^{* * *}$ & $25,70^{* *}$ & $313,01^{* * *}$ & $6,90^{* *}$ & $8,61^{* * *}$ & $443,49^{* * *}$ \\
Propiverin & $76,59^{* * *}$ & $19,25^{*}$ & $236,56^{* * *}$ & $4,43^{* *}$ & $5,63^{* *}$ & $342,47^{* * *}$ \\
Darifenacin & $93,73^{* * *}$ & $39,74^{* *}$ & $321,14^{* * *}$ & $10,47^{* * *}$ & $9,39^{*}$ & $474,48^{* * *}$ \\
INK-Patienten gesamt & $96,07^{* * *}$ & $51,42^{* * *}$ & $362,18^{* * *}$ & $5,92^{* * *}$ & $14,12^{* * *}$ & $529,71^{* * *}$ \\
\hline
\end{tabular}


Tab. 9 Zusätzliche Kosten von OAB- und INK-Patienten in Euro: Hilfsmittel

\begin{tabular}{|c|c|c|c|c|c|c|c|c|c|c|}
\hline \multirow[t]{2}{*}{ Wirkstoff } & \multicolumn{4}{|c|}{ Hilfsmittel bei Frakturen } & \multicolumn{5}{|c|}{ Hilfsmittel hinsichtlich der OAB } & \multirow[t]{2}{*}{ Gesamt } \\
\hline & A 05 & A 10 & A 18 & A 23 & A 11 & A 15 & A 33 & A 51 & A 54 & \\
\hline \multicolumn{11}{|l|}{ Zusätzliche Kosten $O A B$} \\
\hline Solifenacin & $6,13^{* * *}$ & $3,06^{* * *}$ & $21,95^{* * *}$ & $5,73^{* * *}$ & 0,31 & $13,59^{* * *}$ & $0,78^{* * *}$ & $0,17^{* *}$ & 0,46 & $52,17^{* * *}$ \\
\hline Oxybutynin & $4,09^{* * *}$ & $2,85^{* * *}$ & $61,85^{* * *}$ & $21,39^{* * *}$ & $4,80^{* * * *}$ & $115,04^{* * *}$ & $0,86^{* * *}$ & $0,27^{* * *}$ & $2,59^{* * *}$ & $213,75^{* * *}$ \\
\hline Trospium-Chlorid & $4,23^{* * *}$ & $3,51^{* * *}$ & $44,23^{* * *}$ & $7,02^{* * * *}$ & $5,16^{* * *}$ & $54,55^{* * *}$ & $0,92^{* * *}$ & $0,48^{*}$ & $2,30^{* * *}$ & $122,41^{* * *}$ \\
\hline Tolterodin & $3,55^{* * *}$ & $6,24^{* * *}$ & $53,63^{* * *}$ & $7,86^{* * *}$ & $4,86^{* * *}$ & $100,96^{* * *}$ & $2,03^{* * *}$ & $0,24^{* *}$ & $3,19^{* * *}$ & $182,55^{* * *}$ \\
\hline Propiverin & $3,05^{* * *}$ & $2,10^{* * *}$ & $28,21^{* * *}$ & $15,76^{* * *}$ & $-0,57$ & $62,69^{* * *}$ & $0,34^{* *}$ & 0,07 & $1,00^{* * *}$ & $112,65^{* * *}$ \\
\hline Darifenacin & $6,96^{* * *}$ & $5,37^{* * *}$ & $24,36^{* * *}$ & $5,75^{* * *}$ & $2,76^{*}$ & $45,51^{* * *}$ & $1,40^{* * *}$ & $0,17^{*}$ & $1,31^{* *}$ & $93,59^{* * *}$ \\
\hline OAB-Patienten gesamt & $4,39^{* * *}$ & $3,41^{* * *}$ & $43,24^{* * *}$ & $11,44^{* * *}$ & $3,60^{* * *}$ & $67,88^{* * *}$ & $0,92^{* * *}$ & $0,31^{* * *}$ & $1,99^{* * *}$ & $137,16^{* * *}$ \\
\hline \multicolumn{11}{|l|}{ Zusätzliche Kosten INK } \\
\hline Solifenacin & 2,91 & $3,63^{*}$ & $82,60^{* * *}$ & $16,43^{* * *}$ & $23,94^{* * *}$ & $321,16^{* * *}$ & $3,13^{* * *}$ & $1,52^{* * *}$ & $6,08^{* * *}$ & $461,41^{* * * *}$ \\
\hline Oxybutynin & $-0,89$ & $4,58^{* * *}$ & $231,34^{* * *}$ & $84,82^{* * * *}$ & $34,05^{* * *}$ & $773,88^{* * * *}$ & $2,51^{* * * *}$ & $1,51^{* * * *}$ & $14,11^{* * *}$ & $1.145,89^{* * * *}$ \\
\hline Trospium-Chlorid & $-2,20^{* *}$ & $5,16^{* * *}$ & $197,93^{* * *}$ & $19,74^{* * *}$ & $35,45^{* * *}$ & $462,11^{* * *}$ & $1,29^{* * *}$ & $2,72^{*}$ & $13,47^{* * *}$ & $735,66^{* * *}$ \\
\hline Tolterodin & 1,00 & $6,70^{* * * *}$ & $197,77^{* * * *}$ & $13,91^{* *}$ & $29,06^{* * *}$ & $674,33^{* * * *}$ & $4,23^{* * *}$ & $1,36^{* * *}$ & $13,40^{* * *}$ & $941,75^{* * * *}$ \\
\hline Propiverin & $-2,44^{* *}$ & $-1,35$ & $135,50^{* * *}$ & $80,66^{* * *}$ & $11,21^{* * *}$ & $540,81^{* * * *}$ & $1,36^{*}$ & $0,83^{* * *}$ & $8,75^{* * *}$ & $775,35^{* * *}$ \\
\hline Darifenacin & 1,28 & $6,38^{*}$ & $111,21^{* * *}$ & $10,14^{*}$ & $28,61^{* * *}$ & $392,44^{* * *}$ & $1,97^{*}$ & $0,65^{*}$ & $11,80^{* * *}$ & $564,49^{* * *}$ \\
\hline INK-Patienten gesamt & $-0,95$ & $4,38^{* * *}$ & $183,68^{* * *}$ & $41,58^{* * *}$ & $30,53^{* * *}$ & $549,87^{* * * *}$ & $2,05^{* * *}$ & $1,85^{* * *}$ & $12,35^{* * *}$ & $825,35^{* * *}$ \\
\hline
\end{tabular}

$\mathrm{OAB}=$ überaktive Blase; INK = Inkontinenz; A $05=$ Bandagen; A $10=$ Gehhilfen; A $11=$ Hilfsmittel gegen Dekubitus; A $15=$ Inkontinenzhilfen; A $18=$ Krankentransport; A $23=$ Orthesen/Schienen; A $33=$ Toilettenhilfen; A $51=$ Pflegehilfsmittel zur Körperpflege/Hygiene; A $54=$ Zum Verbrauch bestimmte Pflegehilfsmittel

${ }^{*} p<0,10 ;{ }^{* *} p<0,05 ;{ }^{* * *} p<0,01$; robuste Standardfehler

Mehrkosten auf. Die höchsten zusätzlichen Kosten von rund $€ 158$ fallen dagegen bei OAB-Patienten an, die mit Darifenacin behandelt wurden.

Für Inkontinenzpatienten sind die Heilmittelausgaben im Vergleich zu Patienten mit trockener OAB weitaus höher. Auch hier wird das Ergebnis durch die Physiotherapie getrieben. Die geringsten Kosten in diesem Bereich weisen Patienten auf, die mit Solifenacin behandelt wurden, die höchsten Ausgaben Oxybutynin-Patienten.

\subsection{Hilfsmittelkosten}

Die Folgen der OAB beziehen sich - aus Sicht der gesetzlichen Krankenversicherung - hauptsächlich auf den Hilfsmittelbereich, da als Therapiehilfen bei der nassen OAB vor allem Inkontinenzeinlagen verwendet werden.

Die zusätzlichen Hilfsmittelausgaben für OAB-Patienten betragen für OAB-relevante Hilfsmittelausgaben etwa $€ 137$ (siehe Tab. 9). Dies beinhaltet neben den Kosten für OABHilfsmittel ebenso die Hilfsmittelkosten aufgrund von Stürzen und damit einhergehenden Frakturen.

Insgesamt verursachen Solifenacin-Patienten mit rund $€ 51$ die geringsten, Oxybutynin-Patienten mit etwa $€ 214$ die höchsten zusätzlichen Kosten für Hilfsmittel. Insbesondere die Ausgaben für Inkontinenzeinlagen unterscheiden sich stark zwischen diesen Patientengruppen: Während Patienten, die mit Solifenacin behandelt wurden, nur
$€ 14$ zusätzlich für Inkontinenzeinlagen verbrauchen, sind es bei Oxybutynin-Patienten $€ 115$. Da Inkontinenzeinlagen als Therapiehilfen bei der nassen $\mathrm{OAB}$ verwendet werden, sind Ausgaben in diesem Bereich unmittelbarer Ausdruck der Wirksamkeit der OAB-Wirkstoffe. In diesem Sinne erscheint die Wirksamkeit von Solifenacin deutlich höher als die der anderen Medikamente.

Auch für Hilfsmittel bei Frakturen liegen die zusätzlichen Kosten von Solifenacin-Patienten unterhalb der Kosten bei anderen Wirkstoffen. Dies ist vor allem bei Ausgaben für Krankentransport sowie Orthesen und Schienen der Fall. Auch diese Resultate lassen auf eine höhere Wirksamkeit von Solifenacin schließen.

Beim Kostenvergleich der Inkontinenzpatienten zeigen sich ähnliche Resultate. Die Ausgaben im Bereich Hilfsmittel werden insbesondere durch den Posten Inkontinenzhilfen getrieben. Sowohl hier als auch im gesamten Bereich Hilfsmittel weisen Solifenancin-Patienten die geringsten Kosten auf. Die höchsten Ausgaben verursachten dagegen Patienten, die mit Oxybutynin behandelt wurden. Auch hier könnten die Resultate eine bessere Wirksamkeit von Solifenacin im Vergleich zu Oxybutynin andeuten.

\subsection{Zusammenfassung Kostenvergleich}

Insgesamt verursachten OAB-Patienten im Durchschnitt $€ 782$ höhere Ausgaben als nicht-OAB-Patienten (siehe 
Tab. 10 Zusätzliche Kosten von OAB- und INK-Patienten in Euro: Gesamt

\begin{tabular}{|c|c|c|c|c|c|c|c|c|c|}
\hline \multirow[t]{2}{*}{ Wirkstoff } & \multirow[t]{2}{*}{ Stat.-Kosten } & \multirow[t]{2}{*}{ Arztkosten $^{\mathrm{a}}$} & \multicolumn{3}{|c|}{ Arzneimittelkosten } & \multirow{2}{*}{$\begin{array}{l}\text { Heilmittelkosten } \\
\text { (Physio.) }\end{array}$} & \multirow[t]{2}{*}{ Hilfsmittelkosten } & \multicolumn{2}{|l|}{ Gesamt } \\
\hline & & & $\begin{array}{l}\text { Nur OAB } \\
\text { Med. }\end{array}$ & $\begin{array}{l}\text { Ohne OAB } \\
\text { Med. }\end{array}$ & Gesamt & & & $\begin{array}{l}\text { Ohne OAB } \\
\text { Med. }\end{array}$ & $\begin{array}{l}\text { Mit OAB } \\
\text { Med. }\end{array}$ \\
\hline \multicolumn{10}{|l|}{ Zusätzliche Kosten $O A B$} \\
\hline Solifenacin & 67,64 & 146,65 & 347,27 & 80,48 & 427,75 & 96,68 & 52,17 & 443,62 & 790,89 \\
\hline Oxybutynin & 70,81 & 141,12 & 87,21 & 95,15 & 182,36 & 146,64 & 213,75 & 667,47 & 754,68 \\
\hline Trospium-Chlorid & 89,54 & 135,01 & 129,45 & 115,43 & 244,88 & 145,34 & 122,41 & 607,73 & 737,18 \\
\hline Tolterodin & 192,80 & 150,24 & 351,51 & 104,82 & 456,33 & 142,20 & 182,55 & 772,61 & 1124,12 \\
\hline Propiverin & 65,07 & 131,07 & 211,81 & 71,99 & 283,80 & 98,61 & 112,65 & 479,39 & 691,20 \\
\hline Darifenacin & 90,24 & 168,00 & 324,98 & 147,34 & 472,32 & 157,88 & 93,59 & 657,05 & 982,03 \\
\hline OAB-Patienten gesamt & 86,25 & 140,40 & 182,63 & 101,99 & 284,60 & 134,40 & 137,16 & 600,20 & 782,81 \\
\hline \multicolumn{10}{|l|}{ Zusätzliche Kosten INK } \\
\hline Solifenacin & 94,33 & 126,76 & 191,89 & 222,21 & 414,10 & 223,77 & 461,41 & 1098,16 & 1320,37 \\
\hline Oxybutynin & 220,96 & 139,04 & 143,56 & $-47,84$ & 95,72 & 421,38 & 1145,89 & 2070,83 & 2022,99 \\
\hline Trospium-Chlorid & 205,13 & 130,09 & 210,06 & 9,02 & 219,08 & 404,93 & 735,66 & 1685,87 & 1694,89 \\
\hline Tolterodin & 468,02 & 109,80 & 149,67 & 216,06 & 365,73 & 313,01 & 941,75 & 1982,25 & 2198,31 \\
\hline Propiverin & 209,97 & 102,99 & 118,96 & 144,51 & 263,46 & 236,56 & 775,35 & 1443,83 & 1588,33 \\
\hline Darifenacin & 171,04 & 178,91 & 258,32 & 228,19 & 486,51 & 321,14 & 564,49 & 1493,90 & 1722,09 \\
\hline INK-Patienten gesamt & 217,80 & 130,89 & 180,13 & 58,71 & 238,84 & 362,18 & 825,35 & 1716,35 & 1775,06 \\
\hline
\end{tabular}

$\mathrm{OAB}=$ überaktive Blase; $\mathrm{INK}=$ Inkontinenz

${ }^{a}$ Angenommener Entgeltpunkt von 3,5 Cent

Alle Effekte waren hochsignifikant (signifikant) $p<0,01(p<0,05)$ bei zusätzliche Kosten der OAB (INK)

Tab. 10). Die höchsten zusätzlichen Ausgaben fielen bei OAB-Patienten für Arzneimittel an. Dies liegt zum überwiegenden Teil an der OAB-Medikation selbst. Nachfolgend waren der ambulante Bereich sowie die Bereiche Hilfs- und Heilmittel die größten Kostentreiber. Die geringsten Kosten fielen dabei für Patienten an, die mit Propiverin behandelt wurden, die höchsten Kosten bei Tolterodin-Patienten. Bei Einschränkung der Untersuchung auf die Ausgaben ohne OAB-Medikation (d. h. auf die Ausgabenbereiche, die auch zugleich Ausdruck der Wirksamkeit der einzelnen Wirkstoffe sind) liegen die geringsten zusätzlichen Kosten bei Solifenacin-Patienten. Dies lässt, wie oben schon angedeutet, auf eine höhere Wirksamkeit von Solifenacin schließen. Auch bei einer Einschränkung der Untersuchung auf Ausgaben ohne OAB-Medikation weisen Tolterodin-Patienten die höchsten zusätzlichen Kosten auf. Der am häufigsten verwendete Wirkstoff Trospiumchlorid liegt ohne OABMedikation leicht über, bei Berücksichtigung der Kosten für OAB-Arzneimittel dagegen unterhalb der durchschnittlichen Ausgaben von OAB-Patienten.

Beschränkt man sich bei der Auswertung auf Inkontinenzpatienten zeigt sich, dass Solifenacin-Patienten sowohl ohne als auch mit OAB-Medikation die geringsten Kosten im Vergleich zu Patienten mit trockener OAB aufweisen. Dieses Ergebnis ist insbesondere auf die geringeren Kosten im Bereich Hilfsmittel und hier vor allem auf die Ausgaben für Inkontinenzhilfen zurückzuführen. Die höchsten Ausgaben verursachten Inkontinenzpatienten, die mit Oxybutynin oder Tolterodin behandelt wurden. Auch hier steigern die diesmal besonders hohen Ausgaben im Hilfsmittelbereich das Ergebnis.

\section{Diskussion}

Es gibt nur wenige Studien, die einen reinen Kostenvergleich oder einen Vergleich der Kosteneffektivität für die verschiedenen Wirkstoffe bei der Behandlung der OAB durchführen. Ko et al. [16] nutzen Daten zur Wirksamkeit von verschiedenen Anticholinergika in einer Metaanalyse, wobei die Wirksamkeit durch die Kontinenzrate der Probanden abgebildet ist. Ihre Kostenschätzung berücksichtigt die Medikamentenkosten und die Kosten für die Behandlung von Komorbiditäten (Depressionen, Infektionen der Harnröhre, Hautinfektionen). Ko et al. [16] ermitteln für Solifenacin 5mg sowohl die geringsten Kosten als auch die beste Kosteneffektivität.

Milsom et al. [17] untersuchen die Kosteneffektivität von Solifenacin im Vergleich zu Tolterodin für vier nordeuropäische Länder und gelangen zu dem Schluss, dass der Wirkstoff Solifenacin verglichen mit Tolterodin kosteneffektiv ist. Bei den Kosten der OAB berücksichtigen Mil- 
som et al. [17] die Behandlungskosten, die Kosten für Einlagen und die indirekten Kosten des Produktionsverlusts. Die Effektivität wird über qualitätsadjustierte Lebensjahre (QALYs) gemessen.

Cardozo et al. [18] ermitteln die Kosteneffektivität von Solifenacin im Vergleich zu Fesoterodine, Oxybutynin IR, Propiverin, Tolterodin ER und IR für OAB-Patienten in Großbritannien. Sie verwenden Daten zur klinischen Effektivität der Wirkstoffe aus der Metastudie von Chapple et al. [9]. Der Ressourcenverbrauch wurde über eine Befragung von Experten ermittelt und mit offiziellen Preisen verrechnet. Zusätzlich wurden Nutzengewichte aus der Literatur herangezogen, so dass die gemessene Gesamteffektivität dem QALY-Ansatz entspricht. Der Behandlungserfolg wurde separat für Harndrang, Häufigkeit und Inkontinenz ermittelt. Cardozo et al. [18] finden für einen Simulationszeitraum von einem Jahr, dass Solifenacin die höchsten QALY-Gewinne für alle drei Outcomes aufweist. Zudem ist Solifenacin dominant im Vergleich zu Fesoterodine, Tolterodin ER sowie IR und kosteneffektiv im Vergleich zu Propiverin ER. Solifenacin ist jedoch nicht kosteneffektiv (auf Basis eines inkrementellen Kosteneffektivitätsverhältnisses von 30.000 Pfund/QALY) verglichen zu Oxybutynin IR für die Outcomes Häufigkeit und Inkontinenz.

Die beschriebenen Untersuchungen bieten jedoch keinen Head-to-Head Vergleich der Anticholinergika. Klinische Studien weisen zudem den Nachteil auf, dass sie in einer hochkontrollierten Umgebung stattfinden und damit ihre Ergebnisse nicht notwendigerweise repräsentativ für die praktische Umsetzung einer Behandlung sind. Studien, die auf tatsächlichen Versorgungsdaten (z. B. Versicherungsdaten) basieren und damit auch die realen Kosten widerspiegeln sind hingegen nur sehr eingeschränkt verfügbar.

Hall et al. [19] untersuchen mittels individueller Krankenversicherungsdaten aus den USA die unterschiedlichen Auswirkungen von Tolterodin IR und Oxybutynin IR auf die Behandlungskosten der OAB. Sie können weder einen signifikanten Kostenunterschied zwischen den zwei Arzneimitteltherapien feststellen, noch einen signifikanten Kostenunterschied zwischen OAB-Patienten, die mit und ohne Arzneimittel behandelt wurden. Allerdings ist der Betrachtungszeitraum der Studie von vier Monaten nach Diagnose relativ kurz. Dies führt in der Tendenz zu einer Unterschätzung des Behandlungserfolges (und damit der Kostenreduktion) bei der Pharmakotherapie.

Nitz et al. [20] nutzen ebenfalls retrospektive Individualdaten eines US Krankenversicherers und untersuchen den Effekt von Tolterodin ER, Oxybutynin ER sowie Oxybutynin IR auf die Kosten der OAB. Im Gegensatz zu Hall et al. [19] finden Nitz et al. [20] hochsignifikante Kostenunterschiede zwischen OAB-Patienten und Nicht-OABPatienten für die Behandlung mit den einzelnen Arzneimitteln. Diese lagen pro Patient bei 5.074 US-Dollar (Toltero- din ER), 7.486 US-Dollar (Oxybutynin IR), sowie 14.766 US-Dollar (Oxybutynin ER).

Im Gegensatz zu Hall et al. [19] und Nitz et al. [20] zeigt die hier vorliegende Studie, dass die Kosten der OAB bei Patienten die mit Oxybutynin behandelt wurden, geringer sind als bei Patienten die mit Tolterodin behandelt wurden. Studien die einen Vergleich aller Anticholinergika auf Basis von individuellen Realdaten (Head-to-Head) durchführen oder einen Kostenvergleich der unterschiedlichen Anticholinergika für Deutschland beinhalten, fehlten bisher jedoch gänzlich. Eine direkte Vergleichsmöglichkeit der hier gezeigten Ergebnisse mit den Resultaten der bisherigen Literatur ist daher nicht gegeben.

\section{Schlussfolgerung}

In dieser Studie wurde auf Basis von Krankenkassendaten aus dem Jahre 2009 ein Kostenvergleich verschiedener medikamentöser Therapien der überaktiven Blase aus der Perspektive der gesetzlichen Krankversicherung durchgeführt.

Da die überaktive Blase oft im Zusammenhang mit anderen, häufiger auch schwereren Krankheiten (z. B. Diabetes) auftritt und somit die Hauptdiagnose einer Behandlung häufig nicht auf $\mathrm{OAB}$ lautet, ist eine eindeutige Identifizierung von OAB-Patienten anhand von ICD-Nummern schwierig. Eine Auswertung von Nebendiagnosen gilt zudem als problematisch, da deren Kodierung oftmals über längere Zeiträume „mitgezogen“ werden und somit als wenig belastbar gilt. Diese Studie bezieht sich deshalb auf OAB-Patienten, die sich einer medikamentösen Therapie unterzogen haben und bei denen davon ausgegangen werden kann, dass die Diagnose der überaktiven Blase als gesichert gilt.

Insgesamt wurden von einer großen deutschen Krankenversicherung 24.039 Versicherte (von circa 2,9 Mio. Versicherten) anhand ihrer Arzneimittelverschreibungen als OAB-Patienten klassifiziert. Das entspricht einer Prävalenzrate von weniger als einem Prozent. Bisherige Schätzungen deuten auf eine erheblich höhere Prävalenz der OAB hin. So gehen Studien für Deutschland bei Personen über 40 von einer OAB-Prävalenz zwischen $12,7 \%$ [1] und 18,0\% [2] aus. Unsere Studie spiegelt somit nur einen kleinen Ausschnitt der von OAB betroffenen Personen wider. Dies liegt zum einen an der Einschränkung auf eine medikamentöse Therapie, zum anderen an den verwendeten Daten. Diese beinhalten nur reale Abrechnungen der Krankenkasse; Betroffene, die das Problem der überaktiven Blase gegenüber ihrem Arzt nicht erwähnen oder gar nicht erst zum Arzt gehen, werden hier nicht berücksichtigt.

Insgesamt betragen die zusätzlichen Ausgaben eines OAB-Patienten rund $€ 782$. Bei einer Prävalenzrate von einem Prozent entspräche dies rund $€ 547,4$ Mio. Mehrkosten innerhalb der gesetzlichen Krankenversicherung (bei 
70 Millionen GKV-Versicherten). Bei einer Prävalenzrate von $10 \%$ und entsprechender Behandlung müsste mit einem Kostenaufkommen von rund 5,5 Mrd. Euro innerhalb der GKV gerechnet werden. Bisherige Schätzungen gehen für Deutschland von OAB-Behandlungskosten von 4,0 Milliarden Euro insgesamt und etwa 1,8 Milliarden Euro jeweils innerhalb der GKV und der Pflegeversicherung aus [1].

Angesichts dieser hohen Ausgaben kommt einer kostengünstigen und zugleich effektiven Behandlung der $\mathrm{OAB}$ eine große Bedeutung zu. Insbesondere für die medikamentöse Therapie der überaktiven Blase liegen für Deutschland bislang noch keine Studien vor, die die Kosten der verschiedenen Arzneimittelwirkstoffe miteinander vergleichen (Head-to-Head). Mit der vorliegenden Studie sollte diese Lücke geschlossen werden.

Dafür wurde ein Kostenvergleich von sechs Anticholinergika (Solifenacin, Oxybutynin, Trospiumchlorid, Tolterodin, Propiverin und Darifenacin) durchgeführt und für Alters- und Geschlechtseffekte kontrolliert. Der Kostenvergleich erfolgte spezifisch, $d$. h. es wurden nur OAB-bedingte Kosten berücksichtigt. OAB-Patienten, die mit Propiverin oder Trospiumchlorid behandelt wurden, wiesen die geringsten, mit Tolterodin oder Darifenacin therapierte OABPatienten die höchsten zusätzlichen Ausgaben auf.

Des Weiteren wurde eine Zusatzauswertung der Behandlungskosten für Inkontinenzpatienten im Vergleich zu denen für Patienten mit trockener OAB durchgeführt. Dabei wurden OAB-Patienten, die im Bereich Hilfsmittel Einlagen benötigten, als inkontinent klassifiziert. Inkontinenzpatienten, die mit Solifenacin behandelt wurden, weisen die geringsten zusätzlichen Kosten auf. Die höchsten Ausgaben verursachten hingegen Inkontinenzpatienten, die mit Oxybutynin oder Tolterodin behandelt wurden.

Insgesamt weisen Patienten, die mit Solifenacin behandelt wurden, sowohl in der OAB-, als auch in der Inkontinenzauswertung die geringsten Kosten für Folgeerscheinungen der überaktiven Blase (Frakturen) sowie für Therapiehilfen (Inkontinenzeinlagen) auf. Beides deutet auf eine höhere Wirksamkeit von Solifenacin hin. Insbesondere ältere Wirkstoffe wie z. B. Oxybutynin sind zwar selbst deutlich kostengünstiger, jedoch deutlich kostenintensiver bei den benötigten Therapiehilfen (Oxybutynin-Patienten geben $8,5 \mathrm{mal}$ so viel für Inkontinenzeinlagen aus wie SolifenacinPatienten). Da sowohl eine geringere Sturzgefahr, als auch ein geringer Einlagenverbrauch auch eine geringere Gefahr des Einnässens andeuten, ist davon auszugehen, dass eine Behandlung mit Solifenacin die Lebensqualität der betroffenen Patienten deutlich steigert.

Diese Studie führt lediglich einen Kostenvergleich der verschiedenen Anticholinergika aus Sicht der gesetzlichen Krankenversicherung durch. Es bleibt der künftigen Forschung überlassen, den Nutzen der Patienten aufgrund von höherer Effektivität in die Gesamtbewertung der verschiedenen Anticholinergika mit einzubeziehen.
Danksagung Wir danken Thorsten Göbbels, Dr. Simon Löser und Christoph Rupprecht von der AOK Rheinland/Hamburg für die Unterstützung dieser Studie. Interessenkonflikte: Diese Arbeit wurde finanziell durch die Astellas Pharma GmbH Deutschland gefördert.

Open Access Dieser Artikel unterliegt den Bedingungen der Creative Commons Attribution License. Dadurch sind die Nutzung, Verteilung und Reproduktion erlaubt, sofern der/die Originalautor/en und die Quelle angegeben sind.

\section{Literatur}

1. Klotz T, Brüggenjürgen B, Burkart $\mathrm{M}$, et al. The economic costs of overactive bladder in Germany. Eur Urol. 2007;51:1654-63.

2. Milsom I, Abrams P, Cardozo L, et al. How widespread are the symptoms of an overactive bladder and how are they managed? A population-based prevalence study. BJU Int. 2001;87:760-6.

3. Irwin DE, Mungapen L, Milsom I, et al. The economic impact of overactive bladder syndrome in six western countries. BJU Int. 2008;103:202-9.

4. Stewart WF, van Rooyen JB, Cundiff GW, et al. Prevalence and burden of overactive bladder in the United States. World J Urol. 2003;20:327-36.

5. Reeves P, Irwin D, Kelleher CJ, et al. The current and future burden and cost of overactive bladder in five European countries. Eur Urol. 2006;50:1050-7.

6. Reinke C. Harninkontinenz: Frauen sind öfter betroffen, Männer verschweigen sie häufiger. ARS Medici Dossier. 2009;3:24-30.

7. RKI. Harninkontinenz. Gesundheitsberichterstattung des Bundes. Heft 39, 2009.

8. Chapple CR, Khullar V, Gabriel Z, et al. The effects of antimuscarinic treatments in overactive bladder: a systematic review and meta-analysis. Eur Urol. 2005;48:5-26.

9. Chapple CR, Khullar V, Gabriel Z, et al. The effects of antimuscarinic treatments in overactive bladder: an update of a systemic review and meta-analysis. Eur Urol. 2008;54:543-62.

10. Jonas U. Overactive bladder: what matters to the patient? Eur Urol Suppl. 2007;6:423-4.

11. Wagner TH, Hu TW, Bentkover J, et al. Health-related consequences of overactive bladder. Am J Manag Care. 2002;8:598607.

12. Birnbaum HG, Leong SA, Oster E, et al. Costs of stress urinary incontinence: a claims data analysis. Pharmacoeconomics. 2004;22:95-105.

13. Abrams P, Kelleher CJ, Lindsey AK, et al. Overactive bladder significantly affects quality of life. Am J Manag Care. 2000;6:580 90.

14. Darkow T, Fontes CL, Williamson TE. Costs associated with the management of overactive bladder and related comorbidities. Pharmacotherapy. 2005;25:511-9.

15. Horn M. Überaktive Blase: Wenn plötzlicher starker Harndrang Lebensqualität raubt. MMW CME-Spez. 2006;2:1-5.

16. Ko Yu MS, Malone DC, Armstrong EP. Evaluation of antimuscarinic agents for the treatment of overactive bladder. Pharmacotherapy. 2006;26(12):1694-703.

17. Milsom I, Axelsen S, Kulseng-Hansen S, et al. Cost-effectiveness analysis of solifenacin flexible dosing in patients with overactive bladder symptoms in four nordic countries. Acta Obstet Gynecol Scand. 2009;88:693-9.

18. Cardozo L, Thorpe A, Warner J, et al. The cost-effectiveness of solifenacin vs fesoterodine, oxybutynin immediate-release, propiverine, tolterodine extended-release and tolterodine immediate-release in the treatment of patients with overactive bladder in the UK. Natl Health Serv BJU Int. 2010;106:506-14. 
19. Hall JA, Nelson MA, Meyer JW, et al. Costs and resources associated with the treatment of overactive bladder using retrospective medical care claims data. Manag Care Interface. 2001;14:69-75.
20. Nitz NM, Jumadilova Z, Darkow T, et al. Medical costs after initiation of drug treatment for overactive bladder: effects of selection bias on cost estimates. Am J Manag Care. 2005;11:130-9. 